\title{
BMJ Open Prospective multicentre cross-sectional audit among older Australians accessing health and residential aged care services: protocol for a national advance care directive prevalence study
}

\author{
Karen M Detering (D) , ${ }^{1,2}$ Kimberly Buck, ${ }^{1}$ Marcus Sellars, ${ }^{1}$ Helana Kelly, \\ Craig Sinclair, ${ }^{3}$ Ben White, ${ }^{4}$ Linda Nolte ${ }^{1}$
}

To cite: Detering KM, Buck K, Sellars M, et al. Prospective multicentre cross-sectional audit among older Australians accessing health and residential aged care services: protocol for a national advance care directive prevalence study. BMJ Open 2019;9:e031691. doi:10.1136/ bmjopen-2019-031691

- Prepublication history for this paper is available online. To view these files, please visit the journal online (http://dx.doi. org/10.1136/bmjopen-2019031691).

Received 15 May 2019 Revised 28 September 2019 Accepted 02 October 2019

Check for updates

(C) Author(s) (or their employer(s)) 2019. Re-use permitted under CC BY-NC. No commercial re-use. See rights and permissions. Published by BMJ.

For numbered affiliations see end of article.

Correspondence to

Dr Karen M Detering;

karen.detering@austin.org.au

\section{ABSTRACT}

Introduction Advance care planning (ACP), an ongoing communication and planning process, aims to clarify a person's values and preferences, so these guide decision-making if the person becomes unable to make his or her own decisions. Ideally, ACP results in completion of advance care directives (ACD), documents completed by competent people outlining their values, treatment preferences and/or appointment of a substitute decision-maker (SDM). ACDs are most effective at the point of care, where they can be used to inform treatment decisions. Australian governments fund initiatives and have developed policy to increase ACD completion rates. However, little is known about the prevalence of ACDs at the point of care in Australian health services, making ACP evaluation efforts difficult. This study aims to determine the prevalence of ACDs in records of older people in Australian hospitals, aged care facilities and general practices.

Methods and analysis This is a national multicentre cross-sectional prevalence study in selected aged care facilities, hospitals and general practices. Following a 2017 feasibility study, a new protocol incorporating key learnings was developed. Sites will be recruited via expression of interest process. Health records of people aged $\geq 65$ years, admitted to or attending services on study day(s) will be audited by trained staff from sites. Site-level data will be collected during the expression of interest. The primary outcome is the presence of at least one ACD in the health record. Secondary outcomes include prevalence of other documented outcomes of ACP (by health practitioner(s)/family/SDM), assessment of ACD quality and content and concordance between the person's documented preferences and any medical treatment orders. Individuals and sites characteristics where ACDs are present will be explored.

Ethics and dissemination Protocol approval by Austin Health Human Research Ethics Committee, Melbourne, Australia (reference: HREC/18/Austin/109). Results will be disseminated via peer-reviewed journals and conferences. Participating sites and jurisdictions will receive individualised reports of findings.
Strengths and limitations of this study

- This national multicentre cross-sectional prevalence study aims to determine the prevalence and characteristics of advance care directives (ACD) in Australian residential aged care facilities, hospitals and general practices.

- Trained data collectors from participating sites will conduct the audit of health records to identify ACD.

- This protocol builds on the key findings and learnings from a 2017 pilot feasibility study and includes improvements in the training of data collectors, standardisation of data collection and classification of documents.

- The results of this study will contribute to the methodology of undertaking research into the prevalence of advance care planning (ACP) documentation and will inform ACP implementation strategies and evaluation processes.

- The recruitment strategy for sites, using an expression of interest process, is likely to result in a selection bias towards organisations with an interest in ACP.

\section{INTRODUCTION}

Background

Advance care planning (ACP) is a coordinated communication and planning process that aims to clarify and share a person's values and preferences for medical treatment so as to guide healthcare decision-making should the person subsequently lose the capacity to make or communicate such decisions in the future. ${ }^{12}$ The ultimate goal of ACP is to ensure care received is consistent with the person's known preferences. ${ }^{2}$ ACP has been shown to improve outcomes for patients, their families, healthcare staff and the healthcare system. ${ }^{3-6}$

Ideally, ACP discussions should result in documentation recording a person's values and preferences and/or appointment of a 
substitute decision-maker (SDM) ${ }^{2}{ }^{7}$ Documentation is an important component of ACP. It has been shown to improve outcomes for people and provides information and support for SDMs, caregivers and clinicians who may be required to make treatment decisions on behalf of a person whose decision-making capacity is impaired. ${ }^{28-10}$

The names, scope and legal requirements of ACP documentation vary considerably within Australia and internationally. ${ }^{10-16}$ In Australia, ACP documentation includes advance care directives (ACD), a term encompassing documents recognised by state-based legislation (statutory ACD: preferences for care or statutory ACD: appointment of SDM) or common law (non-statutory ACDs) which are completed and signed by a competent adult. ${ }^{17}$ While the specific execution requirements for these three types of ACDs vary, all must be completed by a person with decision-making capacity and they only come into effect once that capacity is lost. ACP documentation may also be completed on behalf of the person by a health professional or someone else (SDM/family). Additionally, medical treatment orders that describe action to be taken in an emergency, such as 'Goals of Care', 'Resuscitation Plans' and 'Physician Orders for Life-Sustaining Treatment', may also include reference to a person's preferences for care. ${ }^{18-20}$ These are completed and signed by a doctor.

For the potential benefits of ACDs and other documented outcomes of ACP to be realised, it is critical that documentation is accessible at the point of care should the person be unable to participate in decision-making. ${ }^{21}{ }^{22}$ If healthcare providers do not have access to the person's documented preferences when treatment decisions are required, the person may receive care that is inconsistent with their preferences. ${ }^{23}$ In Australian health services, a range of systems are used to store and retrieve ACDs, including service-specific electronic health record systems, scanned medical records, hard-copy files or a combination. ${ }^{23}$ Most health services also now have access to 'My Health Record', a new centralised national e-health record that offers online storage of ACDs. While some services may have mechanisms to elicit existing ACDs on admission, many will rely on the person to provide his or her documentation to relevant providers. ${ }^{23}$

Although support for ACP and ACDs continues to grow in Australian policy and legislative frameworks, ${ }^{8} 172425$ there is a lack of national data on the prevalence, content and quality of ACDs at the point of care in Australian health services. Without this information, governments have limited ability to monitor the effectiveness and impact of ACP policy and programmes, and organisations lack evidence to inform service-level programmes and initiatives to increase ACD uptake.

In 2017, an Australian-first pilot feasibility study was conducted by Advance Care Planning Australia (ACPA) to assess the prevalence of ACDs among older adults accessing Australian health and residential aged care services. ${ }^{26}$ Of 2285 health records audited across 51 sites, $30 \%$ contained at least one ACD. The ACD prevalence was significantly higher in residential aged care facilities $(48 \%)$ compared with hospitals $(16 \%)$ and general practices $(3 \%)$ and varied considerably across participating sites. ${ }^{26} \mathrm{~A}$ further $20 \%$ of people had 'other ACP documentation'. This likely included documents that were not formal ACDs (eg, personal letters) or documentation completed on behalf of the person (eg, by a health professional, a family member or the SDM). However, the exact nature or authorship of this 'other ACP documentation' was unclear. ${ }^{26}$

During 2018-2020, ACPA has been funded by the Australian Government to deliver the National ACD Prevalence Study. This study will build on the findings and methodology of the pilot feasibility study ${ }^{2627}$ to collect a national data set on the prevalence and characteristics of ACDs and other documented outcomes of ACP among older people accessing general practices, hospitals and residential aged care facilities across Australia. The study will provide data to assist organisations and governments understand how well ACP is being implemented in Australia and identify areas for improvement. It will also contribute to the methodology of undertaking research into the prevalence of ACP documentation.

\section{Aims and hypotheses}

The aims of the National ACD Prevalence Study are to:

1. Determine the prevalence of ACDs and other documented outcomes of ACP among people aged $\geq 65$ years accessing Australian general practices, hospitals and residential aged care facilities.

2. Assess the content and quality of identified ACDs.

3. Explore consistency between medical orders and the person's documented preferences for care.

4. Explore the characteristics of individuals and study sites where ACDs are present in the health record.

Based on previous Australian studies, ${ }^{2628} 29$ we hypothesise that the overall prevalence of ACDs will be low, and there will be more non-statutory ACDs and 'statutory ACD: appointment of SDM', than 'statutory ACD: preferences for care'. We expect to find various other ACP documents completed by health professionals, SDMs and/or family. We anticipate that prevalence will be highest in residential aged care facilities and lowest in general practice and expect a wide range of ACD prevalence rates within each sector. We hypothesise that there will be variation in the content and quality of ACDs, and that many ACDs will not meet signing and witnessing requirements specified in jurisdictional legislation ${ }^{811}$ or quality criteria outlined in the Australian National Framework for ACDs. ${ }^{8}$ We hypothesise that where there is an ACD, medical orders will be consistent with the person's preferences. We expect a range of individual and site factors to be associated with the presence of an ACD, including demographic and clinical characteristics and site-reported organisational support for ACP. 


\section{METHODS AND ANALYSIS}

Overview of modifications to the original pilot study protocol The 2017 pilot feasibility study ${ }^{26}$ provided key learnings that have informed the current protocol for the National ACD Prevalence Study. Based on these learnings, a number of modifications were made to the study design, site recruitment processes and information collection, training and support for data collectors, data items and data collection tools. These modifications and associated rationale are outlined in relevant sections below.

\section{Study design}

The National ACD Prevalence Study is a prospective multicentre cross-sectional study consisting of two parts ${ }^{1}$ : sitelevel data, collected during the expression of interest process $^{2}$ and an audit of health records of eligible people accessing those services, conducted by trained staff from participating sites.

The pilot study included a self-report survey of people whose records were included in the audit. ${ }^{27}$ The purpose of this survey was to collect information from the person's perspective, regarding their views about and experience with ACP. Feedback received indicated that the survey substantially increased the time and resourcing required to complete data collection, leading to significant additional burdens on study sites. In response, the survey has been removed from the current protocol. We anticipate that this will enable the participation of a wider range of smaller and regional/rural sites that would not have had the resource capacity to conduct both a health record audit and participant survey.

A first round of data collection for the National ACD Prevalence Study was completed in 2018-2019. One further round of data collection is anticipated in 2020. Further rounds of data collection are likely but will depend on funding availability, which is yet to be confirmed.

Sites will be eligible to participate in more than one round of data collection. However, as the study is cross-sectional rather than longitudinal, it is expected that the proportion of sites who participate in more than one data collection round will be low.

\section{Part 1: study sites}

Study sites will include Australian general practices, public and private hospitals and residential aged care facilities, recruited from all eight Australian states and territories (jurisdictions). A minimum of 24 sites will be included in each round of data collection.

\section{Recruitment}

Sites will be recruited through an expression of interest process, coordinated by ACPA and promoted via state and territory departments of health and stakeholder networks using newsletters and websites. Depending on responses to the expression of interest process for each round of data collection, additional sites may be approached by the project team to promote representativeness across sectors (residential aged care facilities, hospital and general

\section{Box 1 Site eligibility criteria}

General criteria: general practices, hospitals and residential aged care facilities are eligible to apply if they:

1. Are an accredited organisation according to sector requirements.

2. Have the approval and endorsement of their executive team.

3. Can nominate one staff member as the Study Lead (responsible for coordination of the study at the site and will be the key contact person for the research team).

4. Have internet, email and telephone access.

5. Have access to devices for online data collection (eg, computer, laptop or iPad).

6. Have policies in place about privacy and confidentiality.

7. Agree that the information provided in their application will be used to generate a Research Collaboration Agreement if successful and are willing to sign this preferably within 4 weeks of being notified of successful application.

8. Meet all sector-specific eligibility criteria.

Sector-specific eligibility criteria

Hospitals and residential aged care facilities are eligible to apply if they:

1. Expect that their site will have at least 50 patients/residents on the day(s) of the study who are aged 65 years or older and have been admitted for at least 48 hours. *

2. Have a records management system with the ability to extract a list of all admissions of people aged 65 years or older who have been admitted for more than 48 hours on the day(s) of the study.

3. Can nominate up to two additional staff members to collect the data for the study.

4. Are willing to support the Study Lead and data collector(s) to undertake mandatory online training in data collection procedures.

5. Have the capacity to review a minimum of 30 health records. Hospitals will also be required to obtain additional ethics approval and/ or a site-specific assessment at their site, ideally within 6-8 weeks of notification of successful application. ACPA will provide support in obtaining necessary approvals as required.

* A minimum of 30 health records will be randomly selected from eligible records on the day(s) of the study.

General practices are eligible to apply if they:

1. Expect that at least 30 patients aged 65 years or older will attend their practice on the day(s) of the study.

2. Have a records management system with the ability to extract a list of all people aged 65 years or older attending the practice on the day(s) of the study.

3. Can nominate up to two additional staff members to collect the data for the study OR agree to data collector(s) being provided by ACPA.

ACPA, Advance Care Planning Australia.

practice) and jurisdictions. All prospective sites will be required to complete an online expression of interest application (see site-level data collection below). Based on feedback from the pilot study, detailed application guidelines including a set of general and sector-specific eligibility criteria (box 1) were developed.

\section{Site-level data collection}

Section 1 of the expression of interest application assesses site eligibility. Sites must meet all criteria before they can proceed. Sections 2 and 3 of the application collects sitelevel data (table 1). Data items are informed by the pilot study, a literature review of system factors potentially 
Table 1 Site-level data variables

\begin{tabular}{ll}
\hline Category & Variable(s) \\
\hline Demographic information & $\begin{array}{l}\text { Jurisdiction, location, funding (government, not-for-profit, private), record management system } \\
\text { used }\end{array}$ \\
Size of service & Number of beds, number of health staff \\
ACP programme/activity & $\begin{array}{l}\text { Current ACP programme/activity within the service, when this commenced, ACP training } \\
\text { available for staff and how ACP is funded and/or implemented } \\
\text { Presence/absence of ACP policy, consumer resources and ACD templates/forms. } \\
\text { (Sites will be asked to upload their ACP policy documents and/or blank ACD templates/forms } \\
\text { used in their service) }\end{array}$ \\
\hline
\end{tabular}

ACD, advance care directive; ACP, advance care planning.

important for ACP/ACD implementation and uptake, and guidance from the project advisory group. Study sites are also required to nominate a Study Lead and one to three data collectors in the expression of interest application.

\section{Part 2: health record audit}

Audit participants will comprise people aged 65 years or older who are admitted to participating hospitals or residential aged care facilities for at least 48 hours prior to audit, or attending general practices on the nominated day(s) of the study. The requirement for admission for at least 48 hours prior to audit is to ensure adequate time for relevant documentation that have been provided or retrieved from the person, the SDM or another service.

Sites will be required to audit the records of a minimum of 30 and a maximum of 50 eligible people. These lower and upper limits are to ensure that sites audit similar numbers of records, thus allowing for meaningful comparison between sites. In the pilot study, sites were required to audit 50 records. However, feedback during recruitment in the pilot study suggested that this precluded the participation of smaller sites that were unlikely to have at least 50 patients/residents meeting eligibility criteria, particularly in rural or regional areas. Thus, the minimum of 30 record audits was applied in the current study to increase opportunities for smaller services to participate, facilitating greater representativeness among participating sites.

Sites are required to nominate how many records they intend to audit (minimum of 30 and maximum of 50 records) prior to their study day(s).

\section{Record selection}

In hospitals and residential aged care facilities, health records will be randomly selected from a list of all eligible people using a simple randomisation procedure, designed to protect against selection bias. On the first day of the study, the site Study Lead will contact their organisation's Health Information Management team (or similar) to obtain a list of current people who meet eligibility criteria. Each eligible person will be assigned a number chronologically, creating an 'Eligible Records List'. These chronological numbers will be used for randomisation. No identifiable information will be provided.

The Study Lead will then inform ACPA of the total number of eligible records, and the number (30-50 records) they intend to audit. Randomisation will be conducted by an ACPA researcher using a random number generator ('Research Randomizer', www.randomizer. org). Records will be assigned to group 1 (include) or group 2 (do not include) within an 'Allocation List', which will be returned to the Study Lead. The Study Lead will match the 'Allocation List' to their 'Eligible Records List' to determine which files to audit. Group 1 will also contain a supplementary list of 10 records which are to be used (consecutively) as needed if any of the initial list are unavailable (eg, patient discharged).

Feedback from Study Leads involved in the pilot study indicated that the randomisation procedure was difficult to understand and implement in practice. To address these issues, Study Leads will receive specific training in the randomisation process, and be invited to complete a trial randomisation procedure prior to the nominated study date. Detailed instructions are also provided in study manuals and online education.

For practicality purposes, consecutive eligible records will be audited in general practices until the required number has been achieved.

\section{Data collection: training, procedures and support}

Participating sites will identify up to three data collectors (may include Study Lead) to undertake the audit. Study Leads and data collectors are expected to complete compulsory online training (approximately $60 \mathrm{~min}$ ) and will have access to a data collection manual (table 2). ACP legislation in Australia is determined by each jurisdiction, ${ }^{11}$ and therefore jurisdiction-specific manuals will be provided. Researchers based at ACPA will be available via telephone for consultation throughout the study.

Data collection will occur during defined periods. Sites are required to nominate 1-3 consecutive days for data collection. Data collectors will obtain selected paper and/ or electronic records (including My Health Record, if applicable) and attempt to locate relevant documentation within $15 \mathrm{~min}$ of opening the record. This time frame was 
Table 2 National advance care Directive prevalence study: data collection training and manual

\section{Online training}

Key content
ACP and ACD
Overview of the National Advance Care Directive
Prevalence Study
- ACP documentation: definitions, classification
for study
- Review of data items and how to collect data

Mode of delivery

- PowerPoint presentation

- Role plays demonstrating:

- A data collector undertaking the audit and entering data.

- A Study Lead performing the randomisation procedure.

- Interactive discussion regarding:

- Types of ACP documentation.

- Demonstration of how to use flowchart for classification.

- Tips for success

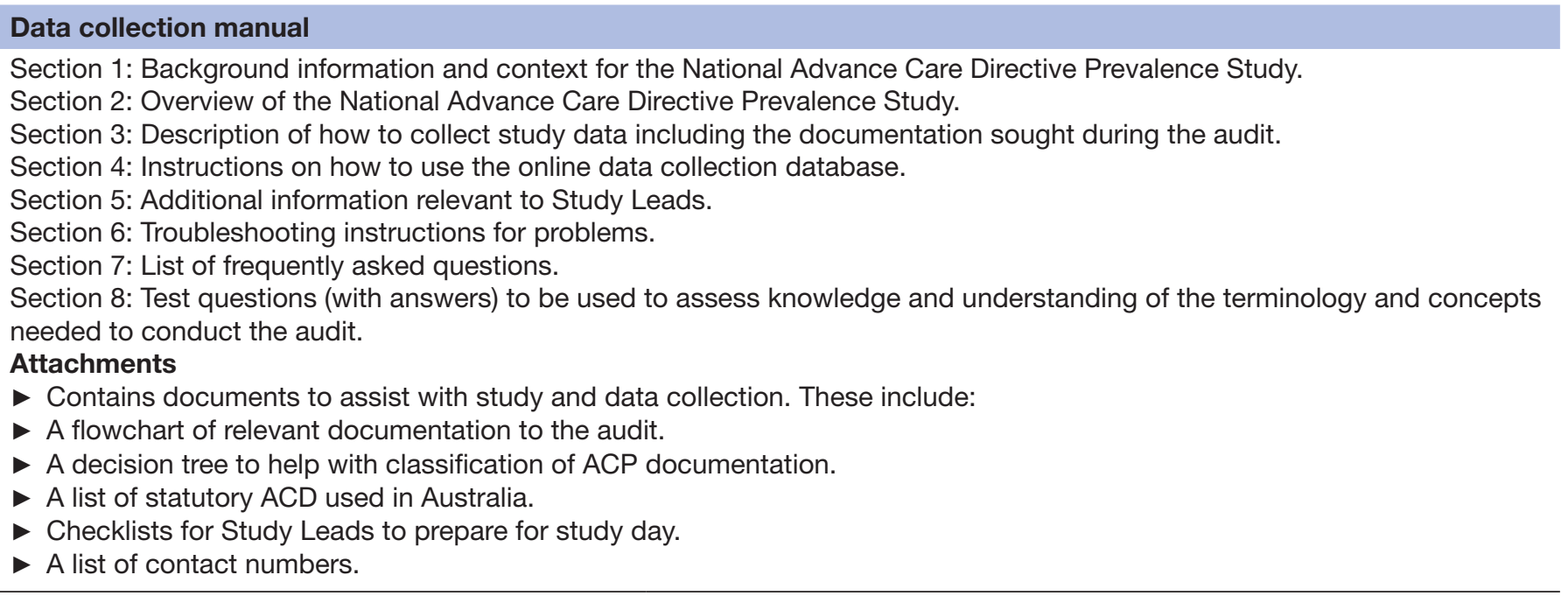

ACD, advance care directives; ACP, advance care planning.

selected in recognition that for ACP documentation and/ or medical orders to be useful, they need to be located quickly. Data variables extracted will include demographic and clinical information, and details regarding the type, content and characteristics of ACP documentation and/ or medical orders (table 3 ).
It is expected that data collection will be completed by staff from participating sites. It is not practical to use external data collectors for a national study of this size. Key learnings from the 2017 pilot study have been used to improve the accuracy of collection. Staff will undertake compulsory training in study methodology and data

Table 3 Participant data variables

\begin{tabular}{ll} 
Category & Variable(s) \\
\hline Demographic information & $\begin{array}{l}\text { Age, gender, postcode, country of birth, relationship status, religion, English or other } \\
\text { language spoken }\end{array}$ \\
Clinical information & $\begin{array}{l}\text { Date of admission/visit, current/active medical conditions (categorised by organ systems), } \\
\text { palliative care status, ECOG status (or estimated functional status if ECOG not available) }\end{array}$ \\
$\begin{array}{l}\text { Type of documentation } \\
\text { (see figure 1) }\end{array}$ & $\begin{array}{l}\text { Completed by: } \\
\text { Classified based on who }\end{array}$ \\
completed documentation & The person (statutory and non-statutory ACD; other, eg, letter). \\
& Someone else (ACP documentation by family, substitute decision-maker, etc). \\
Details of documentation & Miscellaneous ACP material (eg, brochure). \\
& $\begin{array}{l}\text { Time taken to find document, location of document in the health record, date of document, } \\
\text { whether the document contains the person's name, address and date of birth, information } \\
\text { regarding who signed documentation (including witnesses where appropriate), treatment } \\
\text { preferences and/or other preferences specified in the document, characteristics of medical } \\
\text { orders }\end{array}$
\end{tabular}

ACD, advance care directives; ACP, advance care planning; ECOG, Eastern Cooperative Oncology Group. 
collection. Importantly, by supporting staff within organisations to complete data collection, it is anticipated that staff will increase their knowledge and ability to undertake future audits, generating opportunities for implementing ACP initiatives within their services.

ACPA may provide data collector(s) to undertake audit in general practices, and in hospitals and aged care facilities in remote or regional areas that would otherwise be unable to participate due to limited resources. These auditors will not be members of the research team, will complete all required training and will meet privacy and confidentiality requirements of the organisation where they collect data.

All data will be entered directly into a password-protected online database specifically built for this project, and hosted by REDCap (www.project-redcap.org). The database will be available via web-based user interface using a personal computer, laptop or tablet. Each data collector will receive a unique login name and password. Each site will have an identification (ID) number, which will be known only to the site and the researchers conducting the analyses. The database will have in-built validation and range checks to reduce data errors.
The pilot study identified important issues with some data variables. In particular, data collectors reported difficulty classifying ACP documentation. Furthermore, based on free text responses it is likely that some documents were incorrectly classified. Thus in the present study, further information is provided regarding the nature of different documents they may encounter and characteristics of each to facilitate consistent classification. While the pilot study showed ACD prevalence of $30 \%$, a further $20 \%$ of people had 'other ACP documentation'. ${ }^{27}$ The nature of these documents is unclear. ACDs are documents completed by competent people and come into effect if the person subsequently loses capacity to make his or her own decisions. However, ACP documentation is sometimes completed by people other than the person concerned, ${ }^{36212630}$ which may also be of benefit for people without an ACD, who no longer have capacity. This is currently an underexplored area of ACP practice. Consequently, in this study, the process of facilitating correct classification of documentation has changed. We have developed a process and flowchart based on who completed documentation (figure 1) and have provided detailed instructions and examples in education and study manuals.

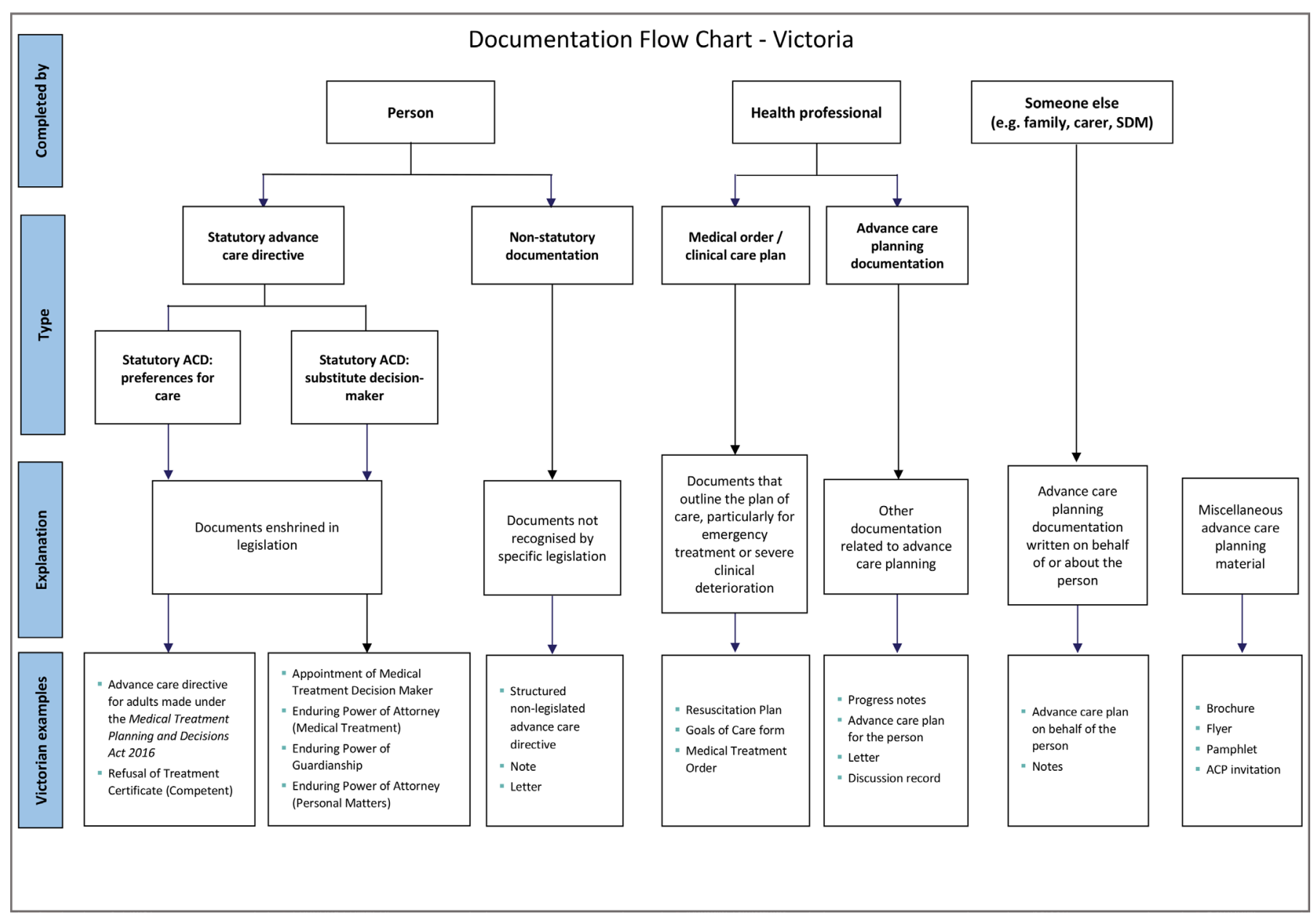

Figure 1 Example of a jurisdiction-specific flowchart provided to data collectors to help them classify documentation identified in the audit. 
Feedback from the pilot indicated that data collectors had difficulty classifying some medical conditions . To address this, data collectors will be instructed to record only current/active medical conditions, and a range of examples will be provided for each of the categories. The pilot also had a substantial amount of missing data (30\%) for the Eastern Cooperative Oncology Group (ECOG) status variable. The revised audit tool includes an additional question asking data collectors to estimate the person's level of functional disability based on available information in the person's record if the actual ECOG rating is not available. These items may be combined during analysis into an overall 'estimated' level of functional disability. In the pilot, there was also evidence of data entry errors in the database. For this study, the database logic has been reviewed, more detailed instructions will be provided within the database and study manuals and additional in-built validation checks will be added. We will also conduct comprehensive user testing of the database and improve education provided to data collectors regarding database usage.

\section{Data de-identification}

A study ID number will be assigned to each person on the audit list, and will be used for data entry. Participating sites will be able to match study IDs with specific people. Identifiable information will not be recorded or disclosed to the research team.

\section{Outcomes}

The primary outcome will be the presence of at least one ACD (statutory ACD: preferences for care, statutory ACD: SDM and/or non-statutory ACD) that is located within 15 min of accessing the record. Secondary outcomes will include prevalence of other documented outcomes of ACP (documents completed by a health practitioner or someone else, such as family or the SDM), assessment of content and quality of ACDs and concordance between the person's preferences for care as documented in their ACD and medical treatment orders. Currently in Australia, there is no standard measure for quality and validity of ACDs. Therefore, documents will be assessed based on requirements specified in jurisdictional legislation, ${ }^{1011}$ and quality criteria outlined in the Australian
National Framework for ACDs, ${ }^{8}$ including whether the document contains the name, date of birth and address of the person, the date of completion, whether the document is signed by the person and/or witnesses and whether any instructions have been provided for the SDM (for statutory ACD: SDM only). To assess concordance between the person's preferences for care and treatment instructions documented in medical orders, the consistency between treatment and/or other preferences specified in the person's ACD will be compared with treatment limitations outlined in their medical treatment order.

\section{Reliability testing}

To estimate any potential bias in rating the primary and secondary outcome variables, two data collectors independently rated the same 40 health records and a reliability analysis was undertaken. Both a percentage agreement and a kappa statistic were calculated for primary (total as well as for each of the three types of ACD) and secondary outcome variables (documentation completed by health professionals and someone else). For each of the primary outcome measures, percentage of agreement between the first and second data collector was 100\% and kappa statistic level of agreement was very high (table 4). For secondary outcome variables, percentage of agreement ranged between $97.1 \%$ and $100 \%$ and kappa statistic level of agreement was good or very good.

\section{Sample size estimation and justification}

Sample size calculations are necessary in prevalence studies to ensure that estimates are obtained with adequate precision. ${ }^{31-33}$ The minimum number of records required for this audit was calculated as 505. This calculation assumes an expected average ACD prevalence of 0.3 (based on pilot study), ${ }^{26}$ confidence level of $95 \%$ and desired precision of $\pm 4 \%$.

While a simple estimate of prevalence shows that a minimum of 505 records are required, to allow for three health sectors to be represented across all eight jurisdictions, 24 sites are required. A minimum sample of 30 records from each of the 24 sites was chosen to minimise the data collection burden, while providing an adequate sample size for site-level results to be reported with a

Table 4 Reliability analysis $(n=40)$

\begin{tabular}{|c|c|c|c|c|}
\hline Variable & $\%$ Agreement & Kappa (95\% Cl) & Kappa agreement & $\mathbf{P}$ value \\
\hline ACP documentation by the person & 100 (5 documents) & 1.0 & Very good & $<0.001$ \\
\hline Statutory ACD: preferences for care & 100 (0 documents) & 1.0 & Very good & $<0.001$ \\
\hline Non-statutory ACP documentation & 100 (2 documents) & 1.0 & Very good & $<0.001$ \\
\hline Documentation by a health professional & $\begin{array}{l}97.1 \text { (33 of } 34 \\
\text { documents) }\end{array}$ & $0.804(0.541$ to 1.0$)$ & Good & $<0.001$ \\
\hline
\end{tabular}

ACD, advance care directive; ACP, advance care planning; SDM, substitute decision-maker. 
precision of $\pm 3.5 \%$. Therefore, the minimum total sample size required will be 720 health records.

\section{Statistical analysis}

Descriptive statistics will be calculated for the total sample and by major grouping site or participant variables. Overall prevalence of ACDs will be calculated for the total sample and separately for healthcare sectors and jurisdictions. Prevalence rates of the types of ACDs, and 'other documentation' will also be reported. Comparisons will be made using t-tests for the continuous type variables and $\chi^{2}$ contingency table analysis for the categorical type variables.

Similar to the pilot study, descriptive statistics will be used to describe the characteristics and content of ACDs and other documented outcomes of ACP (eg, by a health professional or family/SDM), and the specific preferences for care specified in ACDs in comparison the content of medical orders. ${ }^{2627}$

Due to the nesting of individual records within sites, generalised linear mixed model regression will be performed to determine the predictive value of demographic, clinical and site-level variables on the presence of ACD in the person's record. For all analyses, data will be weighted for relevant population characteristics (eg, age, gender, jurisdiction) as necessary, using the latest data from the Australian Bureau of Statistics. Other data sources for weighting such as hospital and aged care demographics will be accessed as required. The level of significance will be set at 0.05 .

If sites participate in more than one data collection round, and there is an analysis that compares prevalence rates over time, then the model will include time, in order to account for repeated measures. If study sample size is insufficient to allow for such a model, data from these returning sites will be limited to the first round of data collection in which they participated.

\section{Project governance}

This study is led by ACPA, and will be overseen by a project advisory group, who will meet approximately four times per year via teleconference. The advisory group is chaired by ACPA, and includes academics and clinicians with interdisciplinary expertise relevant to this study including medicine, nursing, allied health, law and policy. The advisory group also includes representation from hospital, general practice and aged care sectors. The advisory group will co-opt a statistician to provide methodological and statistical advice.

The advisory group will review and endorse the project methodology, and provide advice regarding site recruitment across sectors, risk management, intellectual property and ethical concerns. They will provide advice, drawing on their interdisciplinary expertise, on the collection and interpretation of data and on contextual and jurisdictional aspects of the study.

\section{Confidentiality and privacy}

To avoid any potential breach of confidentiality, only staff of the participating organisations directly involved in collection of data will have access to health records. ACPA auditors will be required to adhere to privacy principles of the services they attend. Data will be handled, stored and disposed off according to the Australian National Health and Medical Research Council Code of Responsible Conduct of Research and the National Statement on Ethical Conduct in Research involving Humans. ${ }^{34} 35$ All results will be de-identified and presented in an aggregate format.

\section{Patient and public involvement}

The specific intent of this project is to inform service-level initiatives and future ACP programmes and policy. People whose records will be audited will not be involved in the audit, the study design or recruitment. As only de-identified information will be collected, it is not possible to provide results to participants. However, study sites will receive de-identified individualised reports including information about their service and how they compare with similar services. The study results will also be provided and discussed with the 'National ACP Engagement Advisory Group', which includes representation from consumers and consumer organisations including National Seniors, Dementia Australia, Palliative Care Australia and the Victorian Cancer Council.

\section{ETHICS AND DISSEMINATION}

Study results will be provided to the participating sites and the Australian Government. No reports will identify any specific participant or site but jurisdictional comparisons will be possible. However, we will exercise caution in reporting jurisdictional or setting prevalence results when such results are primarily driven by only one or two sites.

The results will be highly relevant to clinical practice and policy nationally and internationally; therefore, the findings of this study will also be disseminated through relevant government departments, as well as through various national and international professional bodies, societies and peer-review networks. Findings will be presented at relevant conferences and published in peer-reviewed journals, on the ACPA website and in lay and social media where appropriate. Investigators will review manuscript drafts, abstracts, press releases and any other publications arising from the study. Authorship will be determined in accordance with the International Committee of Medical Journal Editors guidelines.

\section{DISCUSSION}

Effectiveness of national ACD prevalence research requires standardisation of methodology including the accurate classification of ACP documentation, thereby facilitating meaningful measurement and comparison. The current study has built on the findings and key learnings from the pilot feasibility study, ${ }^{26}$ particularly with respect to training of data collectors, standardisation of 
methodology and facilitation of consistency regarding document classification.

\section{Strengths}

The protocol retains the key study design features of the pilot which were shown to be feasible and successful and improves on the identified limitations. The study aims to recruit a diverse range of services increasing the generalisability of the measured outcomes. Data collectors from participating sites are trained in audit methodology and ACD classification using a standardised approach. This should facilitate meaningful comparison of prevalence rates between services. Furthermore, given that data collectors are staff from participating sites, education provided as part of this study has the potential to increase knowledge and awareness at the sites, and therefore contribute to capacity building within these sites.

The National ACD Prevalence Study will generate one of the largest and most comprehensive data sets on ACD prevalence in Australia and internationally. This data set will provide information on the types and characteristics of ACP documentation being used within and across three health sectors that can be used to better understand current practice and inform future ACP strategies and initiatives. The findings will also contribute to the methodology of undertaking research into the prevalence of ACP documentation.

\section{Limitations}

Recruitment via an expression of interest process means that there is a likely selection bias towards sites with an existing interest in ACP. While the aim of this study is to recruit sites across all three health sectors and all eight Australian jurisdictions, it is likely that not all sectors will be similarly represented nor will states and territories, thus limiting generalisability of findings by sector and jurisdiction. As the study focuses on ACD prevalence at the point of care in health and residential aged care services, findings are not generalisable to the wider Australian community. This study uses an audit methodology, and therefore it is not possible to determine quality and extent of ACP conversations known to be an important determinant of a successful ACP programme. Furthermore, given the audit methodology, it will not be possible to determine whether documentation translates into care that is consistent with the person's preferences, the ultimate goal of ACP.

\section{Author affiliations \\ ${ }^{1}$ Advance Care Planning Australia, Austin Health, Heidelberg, Victoria, Australia \\ ${ }^{2}$ Faculty of Medicine, Dentistry and Health Science, University of Melbourne, Melbourne, Victoria, Australia \\ ${ }^{3}$ Centre of Excellence in Population Aging Research, University of New South Wales, Sydney, New South Wales, Australia \\ ${ }^{4}$ Australian Centre for Health Research Law, Faculty of Law, Queensland University of Technology, Brisbane, Queensland, Australia}

Acknowledgements We acknowledge the valuable contribution of the national prevalence study advisory group. We acknowledge the important contribution and feedback provided by Study Leads and Data Collectors from the 2017 pilot study.
Contributors KMD and LN were involved in the conception, design, protocol development and drafting the manuscript; KB was involved in the design, protocol development and drafting the manuscript; MS, HK, BW and CS were involved in the design, protocol development and review of the manuscript. All authors read and approved the final manuscript.

Funding This work was supported by the Australian Government Department of Health.

Competing interests None declared.

Patient consent for publication Not required.

Ethics approval The research protocol for this study was approved on 14 June 2018 by Austin Health Human Research Ethics Committee (reference number: HREC/18/Austin/109). A waiver of consent application was made and granted as part of the original ethics application.

Provenance and peer review Not commissioned; externally peer reviewed.

Open access This is an open access article distributed in accordance with the Creative Commons Attribution Non Commercial (CC BY-NC 4.0) license, which permits others to distribute, remix, adapt, build upon this work non-commercially, and license their derivative works on different terms, provided the original work is properly cited, appropriate credit is given, any changes made indicated, and the use is non-commercial. See: http://creativecommons.org/licenses/by-nc/4.0/.

ORCID iD

Karen M Detering http://orcid.org/0000-0002-1884-7272

\section{REFERENCES}

1 Sudore RL, Lum HD, You JJ, et al. Defining advance care planning for adults: a consensus definition from a multidisciplinary Delphi panel. J Pain Symptom Manage 2017;53:821-32.

2 Rietjens JAC, Sudore RL, Connolly M, et al. Definition and recommendations for advance care planning: an international consensus supported by the European association for palliative care. Lancet Oncol 2017;18:e543-51.

3 Jimenez G, Tan WS, Virk AK, et al. Overview of systematic reviews of advance care planning: summary of evidence and global lessons. $J$ Pain Symptom Manage 2018;56:e25:436-59.

4 Houben CHM, Spruit MA, Groenen MTJ, et al. Efficacy of advance care planning: a systematic review and meta-analysis. J Am Med Dir Assoc 2014;15:477-89.

5 Brinkman-Stoppelenburg A, Rietjens JAC, van der Heide A. The effects of advance care planning on end-of-life care: a systematic review. Palliat Med 2014;28:1000-25.

6 Flo E, Husebo BS, Bruusgaard P, et al. A review of the implementation and research strategies of advance care planning in nursing homes. BMC Geriatr 2016;16:24.

7 Sudore RL, Heyland DK, Lum HD, et al. Outcomes that define successful advance care planning: a Delphi panel consensus. J Pain Symptom Manage 2018;55:e8:245-55.

8 The Clinical Technical and Ethical Principal Committee of the Australian Health Minister's Advisory Council. A national framework for advance care directives. Canberra, 2011.

9 Yadav KN, Gabler NB, Cooney E, et al. Approximately one in three us adults completes any type of advance directive for end-of-life care. Health Aff (Millwood) 2017;36:1244-51.

10 Carter RZ, Detering KM, Silvester W, et al. Advance care planning in Australia: what does the law say? Aust Health Rev 2016;40:405-14.

11 Fountain S, Nolte L, Wills M, et al. Review of advance care planning laws across Australia: short report. Austin Health, Melbourne: Advance Care Planning Australia, 2018.

12 Goffin T. Advance directives as an instrument in an ageing Europe. Eur J Health Law 2012;19:121-40.

13 Weafer JA. A perspective on advance planning for end-of-life. Dublin, Ireland: Irish Hospice Foundation, 2016.

14 Tsoh J, Peisah C, Narumoto J, et al. Comparisons of guardianship laws and surrogate decision-making practices in China, Japan, Thailand and Australia: a review by the Asia Consortium, International Psychogeriatric Association (IPA) capacity Taskforce. Int Psychogeriatr 2015;27:1029-37.

15 Russell S. Advance care planning: whose agenda is it anyway? Palliat Med 2014;28:997-9.

16 Hertogh CMPM. The misleading simplicity of advance directives. Int Psychogeriatr 2011;23:511-5.

17 Australian Commission of quality and safety in health care. The National consensus statement: essential elements for safe and highquality end-of-life care. Sydney: ACSQHC, 2015. 
18 Hickman SE, Keevern E, Hammes BJ. Use of the physician orders for life-sustaining treatment program in the clinical setting: a systematic review of the literature. J Am Geriatr Soc 2015;63:341-50.

19 NSW Government, Office of the Chief Health Officer. Using resuscitation plans in end of life decisions. Available: http://www0. health.nsw.gov.au/policies/pd/2014/pdf/PD2014_030.pdf [Accessed 16 Dec 2014].

20 Sinuff T, Dodek P, You JJ, et al. Improving end-of-life communication and decision making: the development of a conceptual framework and quality indicators. J Pain Symptom Manage 2015;49:1070-80.

21 Hemsley B, Meredith J, Bryant L, et al. An integrative review of stakeholder views on advance care directives (ACD): barriers and facilitators to initiation, documentation, storage, and implementation. Patient Educ Couns 2019;102:1067-79.

22 Buck K, Detering KM, Pollard A, et al. Concordance between selfreported completion of advance care planning documentation and availability of documentation in Australian health and residential aged care services. J Pain Symptom Manage 2019;58:264-74.

23 Detering K M T, Neyland S, Sutton E, et al. Accessibility of advance care plans in Victoria - A summary options paper April 2017. Melbourne, Australia: Department of Health and Human Services, 2017.

24 Victorian Government. Advance care planning: have the conversation. A strategy for Victorian health services 2014-2018. Victoria, Australia: Department of Health, 2014.

25 NSW Ministry of Health. Advance planning for quality care at end of life: action plan 2013-2018, 2013. Available: http://www.health.nsw. gov.au/patients/acp/Pages/acp-plan-2013-2018.aspx [Accessed 8 Jan 2018].
26 Detering KM, Buck K, Ruseckaite R, et al. Prevalence and correlates of advance care directives among older Australians accessing health and residential aged care services: multicentre audit study. BMJ Open 2019:9:e025255

27 Ruseckaite R, Detering KM, Evans SM, et al. Protocol for a national prevalence study of advance care planning documentation and selfreported uptake in Australia. BMJ Open 2017;7:e018024.

28 White B, Tilse C, Wilson J, et al. Prevalence and predictors of advance directives in Australia. Intern Med J 2014;44:975-80.

29 White B, Willmott L, Tilse $\mathrm{C}$, et al. Prevalence of advance care directives in the community: a telephone survey of three Australian states. Intern Med J 2019.

30 Blake M, Doray ON, Sinclair C. Advance care planning for people with dementia in Western Australia: an examination of the fit between the law and practice. Psychiatr Psychol Law 2017:1-22.

31 Arya R, Antonisamy B, Kumar S. Sample size estimation in prevalence studies. Indian J Pediatr 2012;79:1482-8.

32 Hajian-Tilaki K. Sample size estimation in epidemiologic studies. Caspian J Intern Med 2011;2:289-98.

33 Pourhoseingholi MA, Vahedi M, Rahimzadeh M. Sample size calculation in medical studies. Gastroenterol Hepatol Bed Bench 2013;6:14-17.

34 National Health and Medical Research Council. Australian code for the responsible conduct of research. Canberra: Australian Government, 2007.

35 National Health and Medical Research Council. National statement on ethical conduct in research involving humans. Available: https:// www.nhmrc.gov.au/guidelines-publications/e72.2007 [Accessed Mar 2014]. 